\title{
On Positive Functionals on Algebras of Test Functions for Quantum Fields
}

\author{
G. LASSNER* \\ A. UHLMANN** \\ Laboratory of Theoretical Physics \\ Joint Institute for Nuclear Research \\ Dubna, USSR
}

Received July 5, September 15, 1967

\begin{abstract}
It is shown that the *-algebra $R$ of test-functions for a quantum field is reduced, i. e. for each $b \in R, b \neq 0$, there exists a positive continuous linear functional $W(a)$ on $R$ with $W(b) \neq 0$.
\end{abstract}

\section{Introduction}

In the Wightman axiomatical approach a quantum field is defined by a continuous representation of a *-algebra of test-functions in an algebra of (unbounded) operators in a Hilbert space with the same invariant domain and a cyclic vector (vacuum). By the Gelfand-Segal-Theorem a continuous cyclic representation of a $*$-algebra is given by a continuous linear positive functional $W(a)$ on $R$, i.e. a continuous linear functional for which $W\left(a^{*} a\right) \geqq 0, a \in R$, holds. For a quantum field the positive functional $W$ satisfies certain further conditions, i.e. Lorentz invariance, spectrality and locality. Such a functional is called Wightman-functional [1], [2].

The mathematical structure of such a $*$-algebra is described in the following section.

In this paper it is proved that there exists a set $F$ of positive continuous functionals on $R$, which all are bounded by one continuous norm on (the linear space) $R$ so that for every $b \in R, b \neq 0$, there exists a $W \in F$ with $W(b) \neq 0$ and consequently, the algebra $R$ is reduced [3].

It is not proved that there exist "sufficient many" Wightmanfunctionals, but one may hope that the proved result is a step to the solution of this problem.

* On leave of absence from Mathematisches Institut, Karl-Marx-Universität Leipzig.

** Permanent address: Theoretisch Physikalisches Institut, Karl-Marx-Universität Leipzig. 


\section{The *-algebra $\boldsymbol{R}$}

Let $M$ be a topological space ( $M$ is the Minkowski space or the mass shell, for example) and $M^{(n)}=M x \ldots x M$ the Cartesian product of $n$ copies of $M . C\left(M^{(n)}\right)$ is the normed linear space of the continuous complex-valued bounded functions $a_{n}\left(x_{1}, \ldots, x_{n}\right) x_{i} \in M$, on $M^{(n)}$ with the norm $\left\|a_{n}\right\|_{0}=\sup _{x_{1}, \ldots, x_{n} \in M}\left|a_{n}\left(x_{1}, \ldots, x_{n}\right)\right|$. Let $R_{0}$ be the complex field $C$ and for $n=1,2, \ldots R_{n}$ a locally convex linear topological space (over the complex field) of continuous complex-valued bounded functions on $M^{(n)}$ with a stronger topology as is determined by the norm \|\|$_{0}$, i.e. $\left\|a_{n}\right\|_{0}$, $a_{n} \in R_{n}$, is a continuous function on the topological space $R_{n}$.

Furthermore we assume that for $a_{n}\left(x_{1}, \ldots, x_{n}\right) \in R_{n}, b_{m}\left(x_{1}, \ldots, x_{m}\right) \in R_{m}$ $\bar{a}_{n}\left(x_{n}, \ldots, x_{1}\right)$ is an element of $R_{n}$ and

$$
c_{n+m}\left(x_{1}, \ldots, x_{n+m}\right)=a_{n}\left(x_{1}, \ldots, x_{n}\right) b_{m}\left(x_{n+1}, \ldots, x_{n+m}\right)
$$

is an element of $R_{n+m}$ and that the so defined mappings from $R_{n}$ onto $R_{n}$ resp. from $R_{n} \times R_{m}$ into $R_{n+m}$ are continuous.

The algebra $R$ is the linear space

$$
R=\bigoplus_{n=0}^{\infty} R_{n} \quad \text { (topological direct sum [4]). }
$$

Consequently, every element $a \in R$ has the form $a=\sum_{n \geqq 0} a_{n}$ $a_{n}=a_{n}\left(x_{1}, \ldots, x_{n}\right) \in R_{n}$ and only for a finite number of indices is $a_{n}$ different from zero. $a_{n}$ is called the homogeneous component of the degree $n$ of $a$.

The multiplication for two elements $a, b \in R$ is defined by

$$
(a b)_{n}\left(x_{1}, \ldots, x_{n}\right)=\sum_{\substack{k+l=n \\ k, l \geqq 0}} a_{k}\left(x_{1}, \ldots, x_{k}\right) b_{l}\left(x_{k+1}, \ldots, x_{n}\right)
$$

(the product on the right-hand side is the usual product of functions) and the $*$-operation is defined by

$$
\left(a^{*}\right)_{n}\left(x_{1}, \ldots, x_{n}\right)=\bar{a}_{n}\left(x_{n}, \ldots, x_{1}\right)
$$

(the bar on the right-hand side labels the complex conjugate function).

In [1], [2] is $R_{n}=\mathscr{D}\left(M^{(n)}\right)$ resp. $\mathscr{S}\left(M^{(n)}\right)$ the well known Schwartz' spaces of test-functions, but other spaces are regarded in the quantum field theory, too [5]. Here $M$ is the Minkowski space.

Let $K_{0}$ be the algebraical convex cover of the set of elements $a^{*} a$, $a \in R$. Each element $k \in K_{0}$ has the form

$K_{0}$ is a convex cone, i.e.

$$
\begin{aligned}
& k=\sum_{i=1}^{N} a^{(i) * a^{(i)}}, \quad a^{(i)} \in R \\
& a^{(i)}=\sum_{n \geqq 0} a_{n}^{(i)}\left(x_{1}, \ldots, x_{n}\right)
\end{aligned}
$$


a) for $k, k^{\prime} \in K_{0}$ and two arbitrary positive numbers $s, t$ is $s k+$ $+t k^{\prime} \in K_{0}$ and

b) if $k \in K_{0}, k \neq 0$, then $-k \notin K_{0}$.

The statement a) follows directly from the definition of $K_{0}$ and the statement b) holds, because for each $g \in K_{0}$

i) the homogeneous component $g_{r}$ of $g$ with the smallest degree, which does not vanish identically, has an even degree, i.e., $g_{r}=g_{2 s}$

ii) $g_{r}=g_{2 s}\left(x_{1}, \ldots, x_{2 s}\right)$ is nonnegative on the set

$$
\Gamma_{2 s}=\left\{x=\left(x_{1}, \ldots, x_{2 s}\right) ; x_{1}=x_{2 s}, x_{2}=x_{2 s-1}, \ldots, x_{n}=x_{n+1}\right\}
$$

iii) for at least one $\stackrel{x}{\in} \in \Gamma_{2 s}$ we have $g_{2 s}\left(\grave{x}_{1}, \ldots, \grave{x}_{2 s}\right)>0$.

Now we define for $k \in K_{0}$

$$
l_{n}=\sup _{x_{1}, \ldots, x_{n} \in M}\left(\left|a_{n}^{(1)}\right|^{2}+\cdots+\left|a_{n}^{(N)}\right|^{2}\right)^{1 / 2}, \quad n=0,1,2, \ldots .^{1}
$$

Lemma 1. For an arbitrary $k \in K_{0}$ the following relations hold $\left(k_{n}\right.$ is the homogeneous component of the degree $n$ of $k$ ):

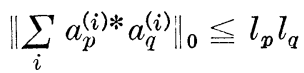

$$
\begin{aligned}
& \left\|\sum_{i} a_{n}^{(i) *} a_{n}^{(i)}\right\|_{0}=l_{n}^{2} \\
& \left\|k_{n}\right\|_{0} \leqq \sum_{\nu=0}^{n} l_{n-\nu} l_{v} \\
& l_{n}^{2}-2 \sum_{\nu=1}^{n} l_{n+\nu} l_{n-v} \leqq\left\|k_{2 n}\right\|_{0}, \quad n=0,1,2, \ldots \quad\left(l_{-1}=0\right) .
\end{aligned}
$$

Proof. (7) follows immediately from the Cauchy-Schwarz inequality by the definitions (2) and (6). Furthermore, we have

$$
\begin{aligned}
\left\|\sum_{i} a_{n}^{(i) *} a_{n}^{(i)}\right\|_{0} & =\sup _{x_{1}, \ldots, x_{2} n}\left|\sum_{i} \bar{a}_{n}^{(i)}\left(x_{n}, \ldots, x_{1}\right) a_{n}^{(i)}\left(x_{n+1}, \ldots, x_{2 n}\right)\right| \geqq \\
& \geqq \sup _{x_{1}, \ldots, x_{n}} \mid \sum_{i} \bar{a}_{n}^{(i)}\left(x_{1}, \ldots, x_{n}\right) a_{n}^{(i)}\left(x_{1}, \ldots, x_{n}\right)=l_{n}^{2}
\end{aligned}
$$

and from this, together with (7) for $n=m$, follows (8). (9) follows from (7) by summing over all $p, q, p+q=n$. From the definition of $k_{2 n}$ we obtain

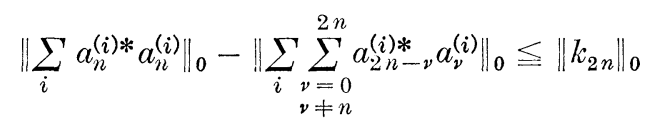

and from this follows (10) by (7) and (8).

1 The application of this expression has been proposed by T. Görnitz, KarlMarx-Universität Leipzig. 
We need further a relation for a special infinite hermitian matrix $H$, which is defined for a sequence $\alpha_{0}, \alpha_{1}, \ldots$ of positive numbers by

$$
\begin{gathered}
H=\left(h_{i j}\right)_{i, j=0,1}, \ldots \\
h_{r r}=\alpha_{r}, h_{i j}=\left\{\begin{array}{l}
-\alpha_{r}, \text { if } i \neq j \text { and } i+j=2 r \\
0, \text { if } i \neq j \text { and } i+j \text { is an odd number }
\end{array} .\right.
\end{gathered}
$$

Lemma 2. There exists such a sequence $\alpha_{0}, \alpha_{1}, \ldots$ of positive numbers that for an arbitrary infinite vector $l=\left(l_{0}, l_{1}, \ldots\right)$ for which only a finite number of components are not zero the relation

holds.

$$
\sum_{i, j \geqq 0} h_{i j} l_{i} l_{j} \geqq \sum_{i} l_{i}^{2}
$$

Proof. We construct by induction a sequence of positive numbers $\alpha_{0}, \alpha_{1}, \ldots$ such that

$$
\sum_{i, j=0}^{m} h_{i j} l_{i} l_{j} \geqq c_{m} \sum_{i=0}^{m} l_{i}^{2}, \quad m=0,1, \ldots
$$

holds, with certain numbers $c_{m}>1$.

For $m=0$ we can set $\alpha_{0}=2$. Now we assume that (13) holds for $m=n-1$ and show that we can choose $\alpha_{0}$ so that (13) holds for $n$, too, with a certain $c_{n}>1$. From the definition of $H$ we obtain

$$
\sum_{i, j=0}^{n} h_{i j} l_{i} l_{j}=\sum_{i, j=0}^{n-1} h_{i j} l_{i} l_{j}+\alpha_{n} l_{n}^{2}-2 \sum_{\nu \geqq 1} \alpha_{n-v} l_{n} l_{n-2 \nu} .
$$

The sum on the right-hand side runs over all $v$ for which the other indices are nonnegative.

From this and the induction assumption we obtain with an arbitrary positive $\beta$

$$
\begin{aligned}
\sum_{i, j=0}^{n} h_{i j} l_{i} l_{j} \geqq & c_{n-1} \sum_{\nu \geqq 1}\left(l_{n-2 v+1}\right)^{2}+\left(\alpha_{n}-\frac{1}{\beta^{2}}\right) l_{n}^{2}+ \\
& +\left(\beta \sum_{\nu \geqq 1} \alpha_{n-v} l_{n-2 v}-\frac{1}{\beta} l_{n}\right)^{2}+ \\
& +c_{n-1} \sum_{\nu \geqq 1}\left(l_{n-2 v}\right)^{2}-\beta^{2}\left(\sum_{\nu \geqq 1} \alpha_{n-v} l_{n-2 v}\right)^{2} \geqq \\
& \geqq c_{n-1} \sum_{v \geqq 1}\left(l_{n-2 v+1}\right)^{2}+\left(\alpha_{n}-\frac{1}{\beta^{2}}\right) l_{n}^{2}+ \\
& +\left(c_{n-1}-\beta^{2} \sum_{i=0}^{n-1} \alpha_{i}{ }^{2}\right) \sum_{\nu \geqq 1}\left(l_{n-2 v}\right)^{2} .
\end{aligned}
$$

Now we choose $\beta$ such that $c_{n-1}-\beta^{2} \sum_{i=0}^{n-1} \alpha_{i}{ }^{2}>1$ and then $\alpha_{n}$ such that $\alpha_{n}-\frac{1}{\beta^{2}}>1$. 
In this manner we have constructed an $\alpha_{n}$ such that the relation (13) holds for $m=n$, too.

Now let $\alpha_{0}, \alpha_{1}, \ldots$ be a sequence of positive numbers for which the assertion of the preceding Lemma holds. Then we define for $a \in R$

$$
\|a\|_{l}=\sum_{\nu \geqq 0} \alpha_{\nu}\left\|a_{2 v}\right\|_{0}
$$

where \|\|$_{0}$ is the norm in $C\left(M^{(2 v)}\right)$ and $a_{2 v}$ is the homogeneous component of the degree $2 v$ of $a$. \|\|$_{l}$ is a continuous semi-norm on $R$ which gives a continuous norm on $R_{l}=\bigoplus_{\nu=0}^{\infty} R_{2 v}$ (the topological direct sum).

Let \|\|$_{u}$ be another continuous semi-norm in $R$ such that for each $a \in R$ and $r=0,1, \ldots\left\|a_{2 r+1}\right\|_{0} \leqq \mu_{2 r+1}\|a\|_{u}$ holds where $a_{2 r+1}$ is a homogeneous component of $a$ and $\mu_{2 r+1}$ are positive constants. Then \|\|$_{u}$ is a continuous norm in $R_{u}=\bigoplus_{\nu=0}^{\infty} R_{2 v+1}$ and

$$
\|a\|=\|a\|_{l}+\|a\|_{u}, \quad a \in R
$$

is a continuous norm in $R$. Beside the basic-topology we regard in $R$ a second topology which is determined by the norm \|\| . This topology is called the norm-topology or \|\| -topology. With this topology is $R$ a (uncomplete) normed linear space, but not a normed algebra.

In the usual cases, where $M$ is the Minkowski space and $R_{n}=\mathscr{S}\left(M^{(n)}\right)$ or $\mathscr{D}\left(M^{(n)}\right)$, the Schwartz' spaces, the semi-norm \|\|$_{l}$ is Lorentz invariant and consequently, we can choose \|\|$_{u}$ such that the norm \|\| is Lorentz invariant, too.

Now we state and prove the main relation for the proofs of the theorems:

Lemma 3. For every $k \in K_{0}$ holds the relation

$$
\sum_{n \geqq 0} l_{n}^{2} \leqq\|k\|_{\imath}
$$

where $l_{n}$ are the expressions (6).

Proof. We obtain from (10)

$$
\sum_{n \geqq 0} \alpha_{n} l_{n}^{2}-2 \sum_{n \geqq 0} \sum_{\nu \geqq 1} \alpha_{n} l_{n+\nu} l_{n-\nu} \leqq \sum_{n \geqq 0} \alpha_{n}\left\|k_{2 n}\right\|_{0}
$$

and in consequence of the definition (11) of $H$ this is equivalent to

$$
\sum_{i, j \geqq 0} h_{i j} l_{i} l_{j} \leqq \sum_{n \geqq 0} \alpha_{n}\left\|k_{2 n}\right\|_{0} .
$$

From this the relation (16) follows, because $\alpha_{0}, \alpha_{1}, \ldots$ is a sequence for which (12) holds. 


\section{Main Theorems}

After the preparations in the preceding section we state and prove here the main theorems.

Theorem 1. The topological closure $K_{\text {of }} K_{0}$ in $R$ with the normtopology, which is determined by the norm (15), is a cone. Consequently, the topological closure $\bar{K}_{0}$ of $K_{0}$ in $R$ with respect to the direct sum topology is a cone, too, because $\bar{K}_{0} \subset K_{\| \cdot}$.

Proof. We prove that the relations (5) i)-iii) hold for $a g \in K_{\|}$, $g \neq 0$, too.

Let $g \neq 0$ be an element of $K_{\| !}$. Then we can write $g=\sum_{n=0}^{m} g_{n}$, where $g_{n}$ is the homogeneous component of the degree $n$ of $g, g_{n}=0$ for $n>m$. 'There has to exist a sequence $k^{v} \in K_{0}$, with $\left\|k^{v}-g\right\| \leqq 1$ and $\left\|k^{v}-g\right\| \rightarrow 0$ for $v \rightarrow \infty$ and consequently,

$$
\left\|k_{n}^{v}-g_{n}\right\|_{0} \rightarrow 0 \text { for } v \rightarrow \infty, n=0,1, \ldots \text {. }
$$

Each $k^{v}$ has the form $k^{v}=\sum_{i=1}^{x_{v}} \underset{v}{a(i) * \underset{v}{a}(i)}, \underset{v}{a^{(i)}} \in R$. Let $l_{n}^{v}, n=0,1,2, \ldots$, be the numbers $(6)$ of $k^{v}$, then we obtain from (16)

$$
\sum_{n \geqq 0}\left(l_{n}^{v}\right)^{2} \leqq\left\|k^{v}\right\|_{l} \leqq\|g\|_{l}+1
$$

and consequently, the sequence $l_{n}^{v}, v=1,2, \ldots$, is bounded for every $n$. Because $g \neq 0$, there exists one $s \geqq 0$ such that

$$
\lim _{v \rightarrow \infty} l_{n}^{v}=0 \text { for } 0 \leqq n \leqq s-1
$$

$l_{s}^{v}$ does not tend to zero for $v \rightarrow \infty$.

Then follows from (9)

$$
\lim _{\nu \rightarrow \infty}\left\|k_{n}^{v}\right\|_{0}=\left\|g_{n}\right\|_{0}=0 \text { for } \quad 0 \leqq n \leqq 2 s-1
$$

Furthermore one has

$$
\lim _{v \rightarrow \infty}\left\|k_{2 s}^{v}\right\|_{0}=\left\|g_{2 s}\right\|_{0} \neq 0
$$

If this is not true, i.e. $\lim _{\nu \rightarrow \infty}\left\|k_{2 s}^{v}\right\|=0$, then we obtain $\lim _{v \rightarrow \infty} l_{s}^{v}=0$ by $(10)$ and the first assertion of (18), which is in contradiction with the second assertion of (18). (19) and (20) are the assertion (5) i). Finally it remains to prove the statements ii) and iii) of (5).

From (17) and (18) we obtain

for $\nu \rightarrow \infty$, i.e.

$$
\begin{aligned}
\left\|g_{2 s}-\sum_{i} a_{v}^{(i) *} a_{v}^{(i)}\right\|_{0} & \leqq\left\|g_{2 s}-k_{2 s}^{v}\right\|_{0}+\left\|\sum_{i} \sum_{\substack{p=0 \\
p \neq s}}^{2 s} a_{2 s-p}^{(i) *} a_{p}^{(i)}\right\|_{0} \leqq \\
& \leqq\left\|g_{2 s}-k_{2 s}^{v}\right\|_{0}+2 \sum_{p=1}^{s} l_{s-p}^{v} l_{s+p}^{v} \rightarrow 0
\end{aligned}
$$

$$
g_{2 s}\left(x_{1}, \ldots, x_{2 s}\right)=\lim _{v \rightarrow \infty} \sum_{i} \bar{a}_{v}^{(i)}\left(x_{s}, \ldots, x_{1}\right) a_{v}^{(i)}\left(x_{s+1}, \ldots, x_{2 s}\right)
$$

11 Commun. math. Phys., Vol. 7 
(in the \|\|$_{0}$-convergence) and consequently, $g_{2 s}$ is nonnegative on $\Gamma_{2 s}$. Because $l_{s}^{v}$ does not tend to zero, we obtain straight-forward from (21) that $g_{2 s}\left(x_{1}, \ldots, x_{2 s}\right)$ is not identically zero on $\Gamma_{2 s}$. Hence, assertion (5) iii) holds, too. $\bar{K}_{0} \subset K_{\|\|}$holds, because the norm \|\| is continuous in the basic-topology of $R$.

Theorem 2. For each $b \in K_{\|}, b \neq 0$, there exists a positive continuous linear functional $W_{b}(a)$ on $R$ with $W_{b}(b) \neq 0$ and $W_{b}(k) \geqq 0$ for $k \in K_{\text {, }}$, for which $\left|W_{b}(a)\right| \leqq\|a\|, a \in R$, holds. Consequently, the topological *-algebra is reduced (see [3] p. 270).

Proof. Let be $b \in K_{\|\|}, b \neq 0$, then it is $0 \notin b+K_{\|\|}$, because $K_{\| \text {is a }}$ cone. Further let $U=\{u:\|u\|<\delta\}$ be such a neighbourhood of the origin, that $U \cap(b+K)=\theta$ holds. Now we define

and

$$
L=\left\{i\left(k_{1}-k_{2}\right): k_{1}, k_{2} \in K_{\|\|}, i^{2}=-1\right\}
$$

$$
K_{1}=\left\{k+s \cdot b+s \cdot u: k \in K_{n, s}, s, u \in U\right\} .
$$

$L$ is a real linear space in $R$ and $K_{1}$ a cone with the interior point $b$ and we find $L \cap K_{1}=\{0\}$ (the origin). For if $a=i\left(k_{1}-k_{2}\right)=k+s b+$ $+s u \in L \cap K_{1}, k_{1}, k_{2}, k \in K_{\|\|}, u \in U, s \geqq 0$, it follows $a^{*}=-a$, i.e. $k+s b+s u^{*}=-k-s b-s u$ and finally $k+s b-s u_{1}=0$, $u_{1}=-\frac{1}{2}\left(u^{*}+u\right) \in U$. If $s>0$, then it would be $\frac{k}{s}+b \in U$ and this is a contradiction to the construction of $U$. Therefore we have $s=0$ and consequently $k=0$, too, i.e. $a=0$.

Now we use

Lemma 4 (MAzur, S.). Let $K$ be a convex set with a interior point $b$ in a real locally convex space $R$ and $L$ a linear subspace of $R$, in which does not lie an interior point of $K$. Then there exists a linear continuous functional $f(a)$ on $R$ with $f(k) \geqq 0$ for $k \in K, f(b)>0$ and $f(a)=0$ for $a \in L[6]$.

If we regard the algebra $R$ as a linear space over the real field, it follows from Lemma 4 the existence of a real linear functional $f(a)$ on $R$ with $f(a)=0$ for $a \in L, f(k) \geqq 0$ for $k \in K_{1}$ and $f(b)>0$. Then $W_{b}(a)$ $=f(a)-i f(i a)$ is a linear functional on the complex linear space $R$ with $W_{b}(b) \neq 0$ and $W_{b}(k)=f(k)-i f(i k)=f(k) \geqq 0$ for $k \in K_{\|\|}$, because $i k \in L$. $W_{b}(a)$ is a positive functional on the algebra $R$. Evidently, we can choose $W_{b}(a)$ so that $\left|W_{b}(a)\right| \leqq\|a\|$ holds.

From the last property it follows that the set $\left\{W_{b}\right\}$ is bounded in the weak topology in $R^{\prime}$ and consequently, by a well known theorem [4], we obtain the

Corollary. The set $\left\{W_{b}\right\}$ of all these positive linear functionals of Theorem 2 is a relatively compact set in the weak topology in $R^{\prime}$ (the dual space of $R$ ). 


\section{References}

1. Borchers, H. J.: On structure of the algebra of field-operators. Nuovo Cimento 24, 214 (1962).

2. Uhlmann, A.: Über die Definition der Quantenfelder nach Wightman und HaAcr. Wiss. Z. Karl-Marx-Univ. Leipzig 11, 2, 213 (1962).

3. Neumark, M. A.: Normierte Algebren. Berlin: VEB Deutscher Verlag der Wissenschaften 1959.

4. Bourbaki, N.: Espaces vectoriels topologiques, ch. II, IV. Paris: Hermann et Cie 1955.

5. JAFEE, A. M.: High energy behavior in quantum field theory. (preprint) Stanford: SLAB-PUB-249; 1967

6. DAy, M. M.: Normed linear spaces, ch. I, §6. Berlin-Göttingen-Heidelberg: Springer 1958. 